\title{
Evolution of Game Controllers: Toward the Support of Gamers with Physical Disabilities
}

\author{
Dario Maggiorini $^{(凶)}$, Marco Granato, Laura Anna Ripamonti, \\ Matteo Marras, and Davide Gadia \\ University of Milan, 20135 Milan, Italy \\ \{dariomaggiorini, marcogranato, lauraripamonti, \\ davidegadia\}@unimi.it,matteomarras@studenti.unimi.it
}

\begin{abstract}
Video games, as an entertaining media, dates back to the ' $50 \mathrm{~s}$ and their hardware device underwent a long evolution starting from hand-made devices such as the "cathode-ray tube amusement device" up to the modern mass-produced consoles. This evolution has, of course, been accompanied by increasingly specialized interaction mechanisms. As of today, interaction with games is usually performed through industry-standard devices. These devices can be either general purpose (e.g., mouse and keyboard) or specific for gaming (e.g., a gamepad). Unfortunately, for marketing reasons, gaming interaction devices are not usually designed taking into consideration the requirements of gamers with physical disabilities. In this paper, we will offer a review of the evolution of gaming control devices with a specific attention to their use by players with physical disabilities in the upper limbs. After discussing the functionalities introduced by controllers designed for disabled players we will also propose an innovative game controller device. The proposed game controller is built around a touch screen interface which can be configured based on the user needs and will be accessible by gamers which are missing fingers or are lacking control in hands movement.
\end{abstract}

Keywords: Video game $\cdot$ Gaming devices $\cdot$ Input device $\cdot$ Game controller • Physical disability

\section{Introduction}

Video games, as an entertaining media, appeared around 1950. Since then, we have been able to observe their constant evolution in both hardware and interaction capabilities. Today, the role of video games in our lives is changing: they are now regarded as viable digital artifacts to deliver interactive stories, teach new skills (edugames), perform physical exercise (exergames), and much more. While the processing power, storage capabilities, and connectivity of gaming devices made huge leaps forward, the hardware apparatuses that let users interact with a game (namely gaming controllers, or just controllers) had a somewhat slower momentum focusing mainly on ergonomics and converging on - sometime legacy - industry standards. Modern controllers range from general purpose tools, inherited from office automation activities (e.g., mouse and 
keyboard), to gaming specific tools (e.g., gamepads). As it is easy to observe, controllers evolved with limited consideration for gamers with physical disabilities.

A physical disability is defined as a limitation on an individual's physical functioning. This limitation may regard mobility, dexterity or stamina. A gamer suffering from mobility or dexterity limitations may experience issues in interacting with a video game. As an example, the player may not be able to provide specific inputs (or combinations of them) due to inability to press multiple buttons at once or move a finger between two positions in a timely manner. These limitations can make the gaming experience unbalanced at least, if not even completely frustrating [4, 29] for the gamer. As an example, it is almost impossible for a player missing the left hand to effectively use a standard modern gamepad. A keyboard is a more viable solution but, nonetheless, it might put a serious disadvantage on the player. To understand the extent of this phenomenon, just think that many devices in the past had limited consideration even for left-handed players; this can be observed e.g., in the Nintendo Power Glove released in 1989. The Nintendo Power Glove is a device designed for the Nintendo Entertainment System (NES). The user must wear the Power Glove on the right hand where tilt sensors and NES controller buttons are located. As a result, all gaming activity must be performed with the right hand while the buttons must be operated with the left one. As a matter of fact, this is putting a disadvantage on left-handed players.

Nevertheless, in recent years, a number of controllers have been designed for gamers with physical disabilities. These controllers are usually variations of industry standards where innovation is aiming to make standard retail games accessible to disabled players. As a result, an attribute shared by many of these controllers is that they try to make available all standard action in a simplified way rather than actually introducing new interaction models.

In this paper, we tackle on the problem of understanding the evolution of game controllers with respect to the usability by players with physical disabilities. In particular, we focus our study on controllers for players with disabilities in the upper limbs; i.e., players that are missing - or have difficulties using - a hand, or part of it. The innovations introduced by existing controllers are analyzed in order to design and propose a novel gamepad which is accessible to gamers with physical disabilities.

The remainder of this paper is organized as follows: in Sect. 2 we analyze the evolution of mainstream game controllers discussing their accessibility for disabled gamers, while Sect. 3 covers existing scientific literature addressing the problem. Current commercial and academic solutions to support physically impaired gamers are presented in Sect. 4. Section 5 describes our innovative solution: the One-Hand Controller, presenting its hardware, software, and interface architectures. Section 6 concludes the paper and outlines possible future research.

\section{Evolution of Gaming Controller Devices}

As already mentioned in the introduction, game equipment dates back to the early ' 50 s with the invention of the Cathode-ray tube amusement device (1947). In this device, the player was supposed to use knobs in order to control the trajectory of a CRT beam spot. Unfortunately, the Cathode-ray tube amusement device was patented but never 


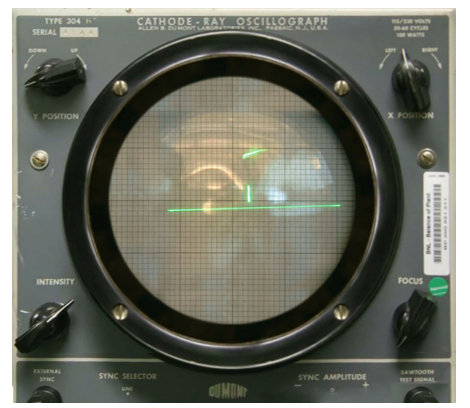

Fig. 1. Tennis for Two reconstruction on a DuMont Lab Oscilloscope Type 304-A.

manufactured or marketed. Other two cases can be mentioned after 1947: Nimrod (1951) and $O X O$ (1952). The Nimrod was a custom-built computer designed to play the game of Nim using a lightbulbs matrix, while OXO was obtained from a repurposed Electronic Delay Storage Automatic Calculator (EDSAC) computer to simulate a game of Noughts and Crosses (usually referenced as Tic-Tac-Toe) on a CRT. Nevertheless, the title of "first video game" is credited to Tennis for Two (1958) by Willy Higinbotham at the Brookhaven National Lab. In Tennis for Two an oscilloscope was used to display a tennis court viewed from the side; players adjusted the angle of their shots with a knob and hit the ball over the net by pressing a button. A reconstruction of Tennis for Two oscilloscope display is proposed in Fig. 1. Anyway, the most important thing for us is that Tennis for Two is reportedly the first gaming equipment using an actual game controller. This game controller, obtained from an aluminum box, was functional but absolutely not comfortable to human hands. A reconstruction of the original controller used by Higinbotham is depicted in Fig. 2. The second game equipped with controllers was Spacewar! (1962). The controllers in Spacewar! were very similar to those in Tennis for Two as form factor but used two double switches rather than a knob. An early design of this controllers is reported in Fig. 3. One important factor here is that, while Tennis for Two (using an oscilloscope) had a requirement for external input devices, Spacewar! ran on a DEC-PDP-1 and could use the console's single toggle switches. Nevertheless, the developers declared that native controls were not adequate for a game [11] and specific external controllers have been designed. This is reportedly the very first example of a game causing the development of a controller; as also discussed in [5].

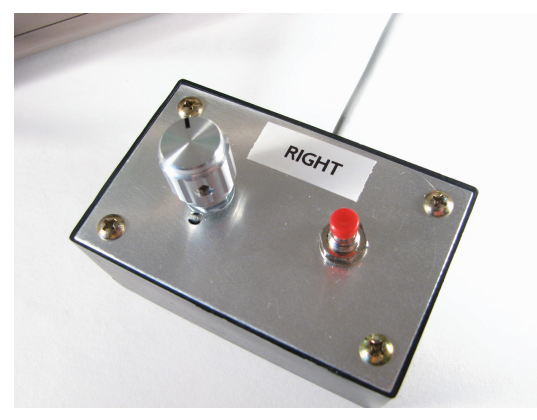

Fig. 2. Reconstruction of Tennis for Two controller. 


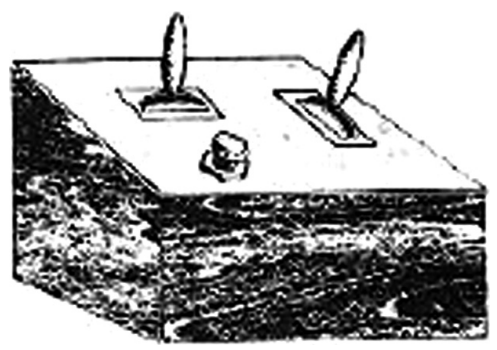

Fig. 3. Spacewar! controller design. Source: Creative Computing Magazine, August 1981.

Anyway, it goes without saying that sharp edged metal boxes were everything but ergonomic to use, let alone that disabled players where not considered at all. Knobs are impossible to manage without a decent finger grip and a push button alone was not a convenient way to play.

We have to wait until the beginning of the ' 70 s to see the appearance of the first coin-operated (or coin-op) machines and gaming consoles. Coin-ops, such as Galaxy Game (1971), Computer Space (1971), and Pong (1972) were intended for public use and adopted controls bolted in the metal frame while gaming consoles like the Magnavox Odyssey (1972) have been marketed for home entertainment and used detachable controllers. Nevertheless, in the majority of the cases, the interaction model was still bound to a rotary wheel and one or two buttons. At this stage, controllers have been rebaptized paddles and improved in form factor. Actually, the form factor has been improved only for non-disabled gamers since paddles need to be held in one hand while operated with the other. The impossibility, in the majority of cases, to use a stable surface as a support further reduced accessibility for disabled players.

In the late ' $70 \mathrm{~s}$, with the second consoles generation, gaming controllers evolved into many shapes with different functionalities. Of those shapes, two are still mainstream today: the joystick and the gamepad. A joystick is an input device based on the design of an aircraft's control system and has been widely associated to Atari's consoles. The joystick proved to be the first truly generic control device fitting a huge number of games. Moreover, its operation proved to be possible also by gamers with sometimes severe - limitations in the upper limbs. As a matter of fact, these same devices are also used to operate assistive medical devices such as motorized wheelchairs. The gamepad, on the other hand, was also introduced by Atari but it has been more largely associated to Nintendo's consoles. The first form of gamepad (also called joypad) featured a cross-shaped $D-P a d$ button to move in four directions and two buttons for interaction; see e.g., Figure 4 . While the joypad proved to be superior to the joystick thanks to smaller movement required to play, its use was still unfriendly to gamers with physical disabilities in the hands and, from their usability standpoint, a step back from the joystick.

After the introduction of joystick and joypad, many other gaming controllers have been proposed. For sake of clarity, and to keep our study focused, in the remainder of this section we will concentrate on gaming controllers to be operated using hands and with a reasonably high market/adoption relevance. 


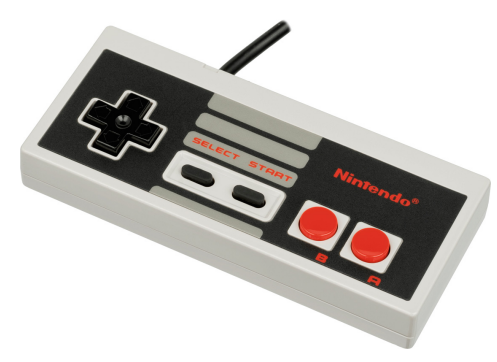

Fig. 4. The first gamepad from Nintendo (the joypad).

The following evolution of game controllers has been triggered by the introduction of $3 \mathrm{D}$ games thanks to the increased processing power available in the fifth consoles generation (1993-1998): the D-Pad, due to its four-directions nature, proved to be inefficient in navigating a 3D environment. As a result, the joystick was merged back to contribute with an analog finely-tuned control. The first console performing this merge back has been the Nintendo 64 (1996), whose controller can be seen in Fig. 5. While using a standard joystick was a feasible task for a disabled gamer, a thumb-sized one to be used in coordination with the D-Pad rolled back the situation to the previous generation of controllers. Mainstream controllers were, once again, extremely difficult to manage for players with disabilities in the upper limbs.

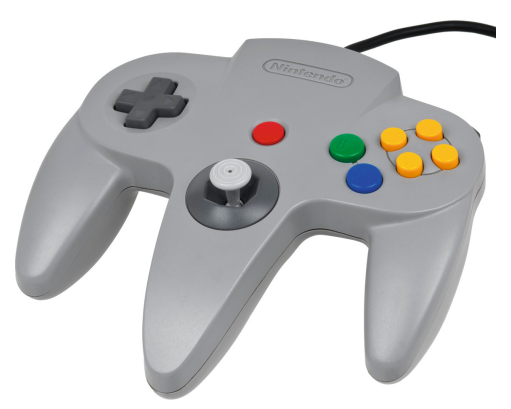

Fig. 5. The Nintendo 64 gamepad.

Gamepads evolution observed just two other major steps forward. Both these improvements took place during the sixth console generation (1993-1998) and consisted in the addition of analog buttons (not used by any modern game actually) and the haptic feedback. Haptic feedback, in particular, is of little interest in the current discussion because it provides an output to the user instead of collecting inputs. As a result, gamepads changed in shape for a more favorable hold (for a non-disabled player) but are keeping today the same functionalities established with the Nintendo 64 . A modern gamepad from Microsoft can be seen in Fig. 6 . 


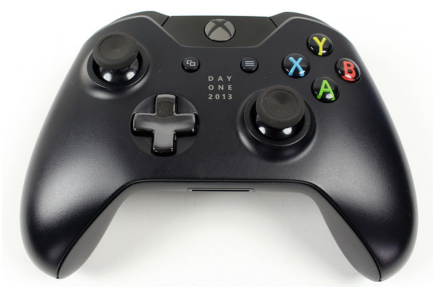

Fig. 6. Microsoft Xbox One controller.

Despite the fact that gamepads did not change much over time, other kinds of controllers showed up in recent years proposing completely innovative interaction models: motion control and computer vision interactivity.

Motion control has been adopted by both the Nintendo Wii and the Sony Playstation 3 consoles. While the Sony Move failed to be a commercial breakthrough due to limited support in mainstream games, the Nintendo Wii Remote and Nunchuck (Fig. 7) proved to be a real gamechanger in the interaction scenario. With the Wii Remote, D-Pad and thumbstick are separated in two controllers linked by a wire and a player can interact with the game by waggling each controller separately. Despite its lack of precision in registering movements, the Wii Remote become extremely popular among players, also because D-Pad and thumbstick, albeit available, did not any longer represent a major way of interaction. This acceptance has been extended to the point to exploit the controller for actual medical rehabilitation programs [10, 30]. Another interesting controller for the Nintendo Wii worth mentioning - despite not being operated with hands - is the Wii Balance Board. The Wii Balance Board used pressure sensors to monitor player's center of balance and weight and was also exploited in a number of rehabilitation programs $[2,18]$. Unfortunately, the Wii Balance Board has not met a huge commercial success. Going back to the Wii Remote, it proved to be very useful to a number of disabled gamers: even when their finger control was limited this controller provided a very enjoyable experience. The same interaction model proposed by the Wii Remote has been kept by Nintendo up to the last console generation, on the Nintendo Switch. The Nintendo Switch features two detachable controllers called Joy-Cons which can be used in a similar way as the Wii Remote and Nunchuck. Despite this, Joy-Cons are smaller than their predecessors and current games require a more intensive use of buttons and thumbsticks, making them less attractive for a player with difficulties in using her hands.

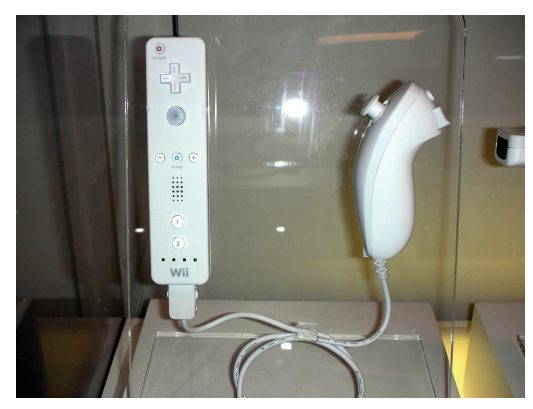

Fig. 7. Wii Remote and Nunchuck. 
Computer vision interactivity uses computer vision technology to analyze images of the player's movements and translate it into movement in the game. This has been attempted initially by Sony with the EyeToy device (2003) (Fig. 8 on the left) on the Playstation 2 and then relaunched by Microsoft with the Kinect (2010) (Fig. 8 on the right), which proved to be a huge commercial success. Like the Wii Remote, the Kinect has also been used in a relevant number of rehabilitation projects; see e.g., [6, 21, 22]. Kinect proved to be a really convenient and accessible device for gamers with disabilities. Nevertheless, it is worth mentioning that this convenience is limited to players suffering from reduced control. The body tracking technology is not currently able to cope with missing body parts and the presence of assistive devices such as wheelchairs. Moreover, we must also point out that limitation in movement (e.g., due to poor control of the lower limbs) and sensibility to physical exertion may put a severe limit to the gameplay.

A device similar in concept to the Kinect but specialized in short range hand tracking is the Leap Motion Controller (2013). The Leap Motion Controller uses infrareds to track finger positions. Unfortunately, this device assumes all fingers are in place when tracking and is not useful for gamers missing one or more.
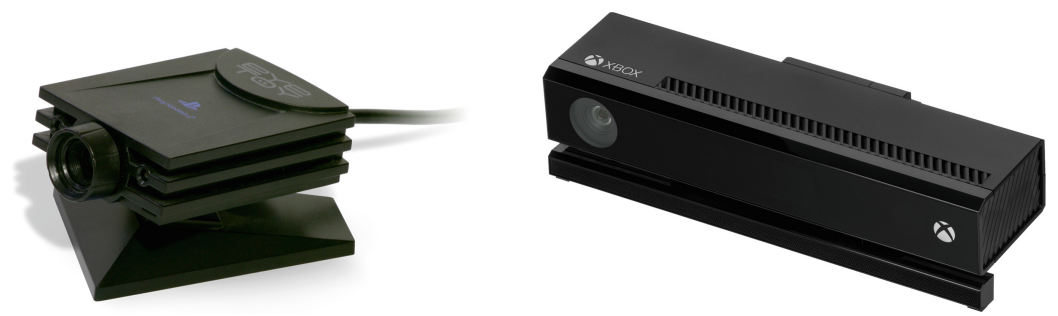

Fig. 8. Computer vision devices: Sony EyeToy (left) and Microsoft Kinect (right).

To close our historical analysis, we must also mention the most used gaming devices of all times: keyboard and mouse. Keyboard and mouse are not used on consoled but get normally adopted by PC gamers. Differently from their consoles' counterpart, these two input devices, probably due to their general-purpose nature, did not change much over the years. Today, it is possible to find many keyboards and mice on the market tailored for users with disabilities. Unfortunately, to the best of our knowledge, none of them are designed with gaming activities in mind. Usually, the addressed impairments are relative to not being able to see or hit the keys correctly when typing while typists with reduced movements can benefit from alternate physical layouts. Usually, a disabled player on PC is inclined to augment her setup with additional devices (e.g., pedals), use software aids (e.g., commands triggered by vocal patterns), or even create her own custom devices. 


\section{Related Work}

Moving now to a more scientific ground, we can observe that in recent literature there is a vivid interest in game accessibility [28, 33, 34]. On this ground, the Independent Game Developers Association (IGDA) published already in 2004 a set of guidelines for gaming accessibility [16]. Unfortunately, these guidelines focused mainly on visual impairments, leaving out gamers suffering from physical limitation due to reduced mobility or missing limbs. The aforementioned guidelines have been updated by [24] in 2006, but their general goal was, unfortunately, not extended. Other interesting sources for accessibility guidelines, created as a collaborative effort with the academia, are represented by the Game Accessibility Guidelines website [9] and the AbleGamers Foundation's Game Accessibility Guidelines [3].

The scientific community is contributing to this topic by proposing innovative devices for disabled gamers with a higher accessibility when compared to ones available on the mass market.

To address the introduction of new devices, the research is mainly focused on making standard controllers available to impaired people through physical adaptation and integration with additional sensors, such as in [7, 15]. Unfortunately, their approach seems to be a bit intrusive and cumbersome to setup for the average player due to the additional wiring and sensors unsupported by the console vendor. Nevertheless, these contributions proved to be an interesting solution to support some cognitive disabilities.

Other researchers are working to completely exclude the physical interaction with a controller. To remove the physical interaction, it is possible to adopt Brain-Computer Interface (BCI) [20] or electromyography (EMG) [17, 32] technologies. Application of these approaches can also be found in commercial products such as the Epoc+ (2013) from Emotiv and the Myo armband (2015) from Thalmic Labs.

The combination of the approaches above, may result in using a sensor system in place of a controller like in the case of the VoodooIO Gaming Kit [31]. In the VoodooIO Gaming Kit, players are allowed to place physical inputs as they need on a conductive fabric.

Alongside new devices, there is also a demand for methodologies on how to integrate them in new or existing games. To this purpose, we are witnessing a significative effort to provide guidelines to increase accessibility through game design.

In [25], we can find a study about making classic games accessible to disabled gamers. In this work, a middleware between the game and the I/O subsystem has been created using a descriptive language. This result aims to demonstrate that - technically - any game can be made compatible with any kind of device. Unfortunately, this approach seems to be too invasive for the game core architecture because it requires the (assistive) I/O device to have a direct API access to the hosting game engine. This requirement translates to the development of a game engine specific to the device and, in turn, to the supported disability. Another contribution along the same line [12] led to the creation of a game that, with specific changes to game mechanic and logic, can be adapted to any kind of player's disability. A very specific contribution is presented in [14], where the design of fast-paced action-oriented games for children with cerebral 
palsy is discussed. In this paper, a participatory design process is used to prove the feasibility of the approach and provide a set of recommendations to achieve actionorientation and playability.

Research on advanced game controllers is also performed outside the scientific community to the purpose of creating commercial products. Unfortunately, none of those reached popularity and become mainstream. Nevertheless, they have all been (and some of them still are) marketed. In the next session we are going to address game controllers for disabled gamers which have been proposed as commercial products.

\section{Commercial Devices Suitable for Players with Disabilities}

To overcome their physical limitations, impaired players usually look for specific input devices. On the market, many devices have been proposed by gaming-oriented companies to improve (or make possible) gaming experience of physically disabled players.

Most probably, the first (and the best) commercial example of a controller to support disabilities is the NES Hands Free Controller (1989) by Nintendo. This controller was designed for gamers totally unable to move the hands and/or a significative part of their body. This device must be strapped to the chest and hooked to the neck of the player (Fig. 9). For movements, the gamer can use her chin to move a joystick while buttons are simulated by blowing in a small pipe.

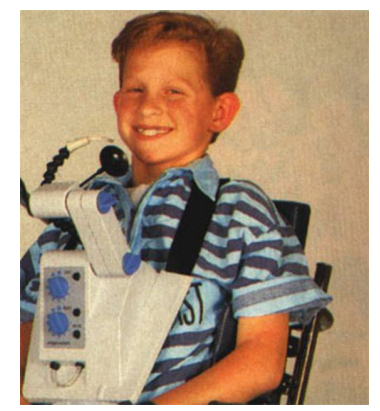

Fig. 9. A Kid wearing the NES Hands Free Controller. Source: [26].

After the NES Hands Free Controller, we have to wait almost ten years to see the ASCII Grip Controller (1996) from Agetec. This controller was originally designed for the fifth generation of game consoles (1993-1998) and conceived to let gamers play using only one hand while merging all interaction under the control of 5 fingers (Fig. 10). The goal of the ASCII Grip Controller was to support disabled gamers by making available all inputs which are present on a standard controller with minimal effort and movement. 


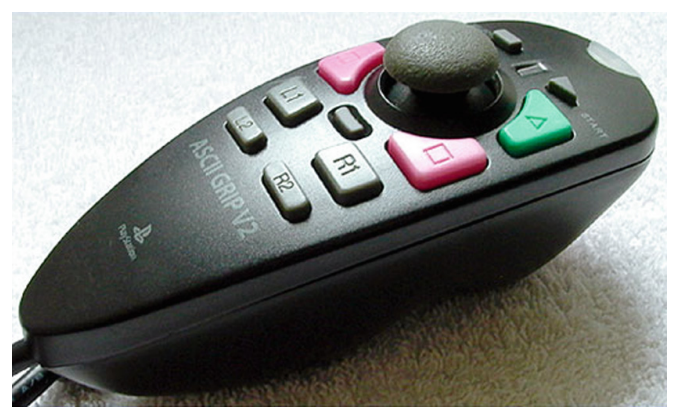

Fig. 10. ASCII Grip Controller. Source: oneswitch.org.uk.

A similar solution to the ASCII Grip is proposed by the DragonPlus DuoCon (2008), where all the controls of a standard gamepad are placed on a tabletop case and the player can rest her hand on top of an ergonomic support. Controls are located at the end of the palm-resting platform to avoid straining (see Fig. 11). As it can be easily observed in the picture, the intended platform this controller was the Sony Playstation 3 (seventh generation). The DragonPlus DuoCon was updated in 2010 and re-baptized One-Handed Ergonomic Palm Game Controller. While the concept and form factor were still the same, this second version further extended consoles compatibility up to Playstation 4 and Xbox One.

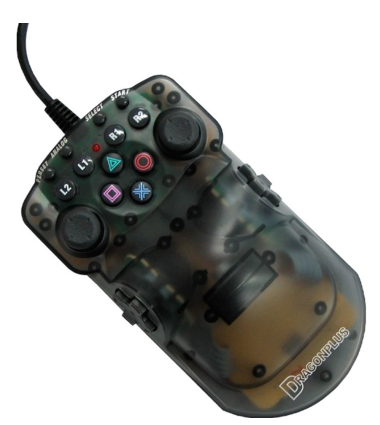

Fig. 11. DragonPlus DuoCon Controller. Source: oneswitch.org.uk.

A very interesting step forward to support one-handed gamers is also represented by the eDimensional Access Controller (2008). As we can see in Fig. 12, like the previous one, also this controller extends the concept of tabletop case but, differently from the DragonPlus DuoCon, it is providing a modular architecture. Controls are divided into independent units and each unit can be plugged in a socket of the main case. By deciding the position of each control unit, a disabled gamer can customize the controller based on her specific disability. 
Alongside with the companies we mentioned in this section, we can observe the constant growth of other organizations focused on helping disabled players. Worth mentioning are OneSwitch and AbleGamers. OneSwich offers an updated and affordable market for enabling gaming solutions, not just controllers. AbleGamers is a nonprofit public charity aiming to improve the overall quality of life for those with disabilities through video games. They create tailored solutions for single users and perform R\&D for assistive gaming devices.

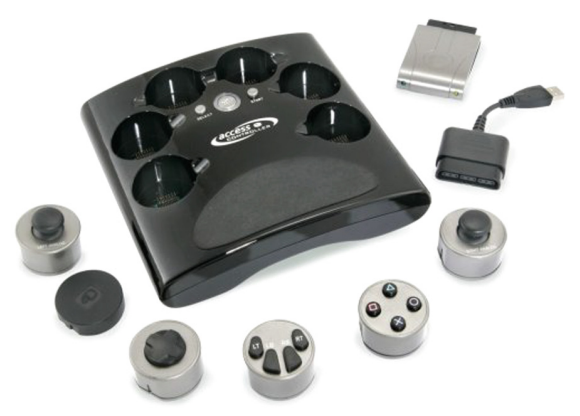

Fig. 12. OHC concept design. Source: [8].

\section{OHC: A Novel and Accessible Gamepad}

Designing a game or a device to cope with all possible disabilities is very difficult, but not impossible, as discussed by [25]. As already mentioned in the introduction, in this manuscript we are going to focus on gamers suffering from mobility issues in the upper limbs. In particular, our research targets players which are missing one hand (or part of it) or have severe limitations in the mobility of their dominant hand.

Starting from the above considerations and taking inspiration from the analysis we performed in the previous three sections, our guidelines can be summarized as follows:

- an analog joystick-like control is a requirement;

- the controller should not be held in one hand, but must lay flat on a supporting surface;

- controls should be configurable in order to adapt to each disability;

- compatibility with standard USB Host-Computer Interface must be granted in order to cooperate with other commercial products.

Working with the above guidelines, we designed $\mathrm{OHC}$ : One-Hand Controller. OHC leverages on a mix of analog and digital inputs. Analog inputs are collected by means of a physical thumbstick while buttons are emulated using a touch interface. This touch interface is positioned flat on a supporting surface and the controls it represents can be customized by the player in layout, orientation, and size. We selected a touch interface because of three important advantages over current hardware solutions. First, it allows a flexible and fine-tuned re-configuration of gaming controls. Second, it might be feasible to substitute the touch surface with a tablet or smartphone already owned by 
the player, thus achieving a consistent cost reduction. Last, as an ongoing project, a touch interface allows fast prototyping of controls layouts and easy data collection about hand posture and touch patterns. A concept design of $\mathrm{OHC}$ can be seen in Fig. 13. As it can be observed, the device is intended to be completely symmetrical; this way it can be easily flipped to support both right- and left-handed players.

The OHC controller has already been introduced in [8], in the following subsections we are going to describe its updated hardware and software architectures and will propose a new set of experiments to evaluate user acceptance rate.

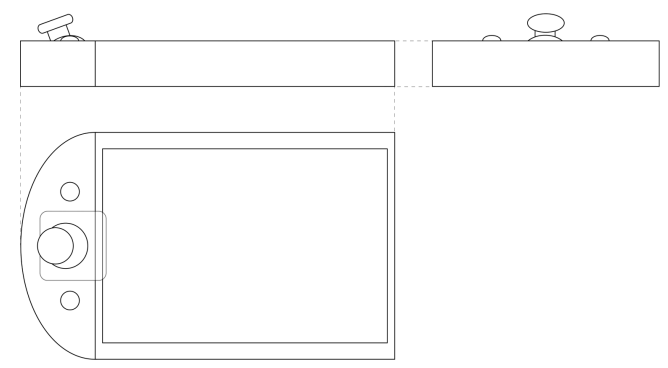

Fig. 13. eDimensional Access Controller. Source: oneswitch.org.uk.

\subsection{OHC Hardware Architecture}

OHC is built around a 7" LCD multi-touch touchscreen equipped with an analog stick and two pushbuttons (provided for configuration purposes). The touchscreen supports up to five fingers at once and the analog stick is two-axis thumbstick. The controller hardware is managed by two interoperating microcontrollers: a Raspberry PI3 and an Arduino Leonardo. A scheme of the hardware setup is reported in Fig. 14.

The Raspberry PI microcontroller is in charge to drive the LCD touchscreen using the onboard Display Serial Interface (DSI). Using the DSI, the 7" LCD can be driven at 25 frames per second. The Arduino Leonardo alone would not provide enough bandwidth to drive such a large display. Moreover, the Raspberry is also in charge to collect user inputs. These user inputs include both touches on the screen and the external thumbstick with pushbuttons. Unfortunately, there are no analog inputs on the Raspberry GPIO bus. Therefore, we were forced to use an Analog to Digital Converter (ADC) to convert the thumbstick position. To perform this conversion, we used an MCP3008, but any 10 bits converter supporting $5 \mathrm{~V}$ digital outputs can be used.

The Arduino Leonardo oversees managing the communication between $\mathrm{OHC}$ and the gaming PC. Leonardo has been used since it natively supports USB slave mode, and libraries to emulate mouse and keyboard are ready available. This way, the Raspberry PI can decode user input and use the Arduino to remap the result to legacy inputs for the game. Raspberry PI alone is not able to perform this task because it - as a system-on-chip computer - can only work as a USB Host. 


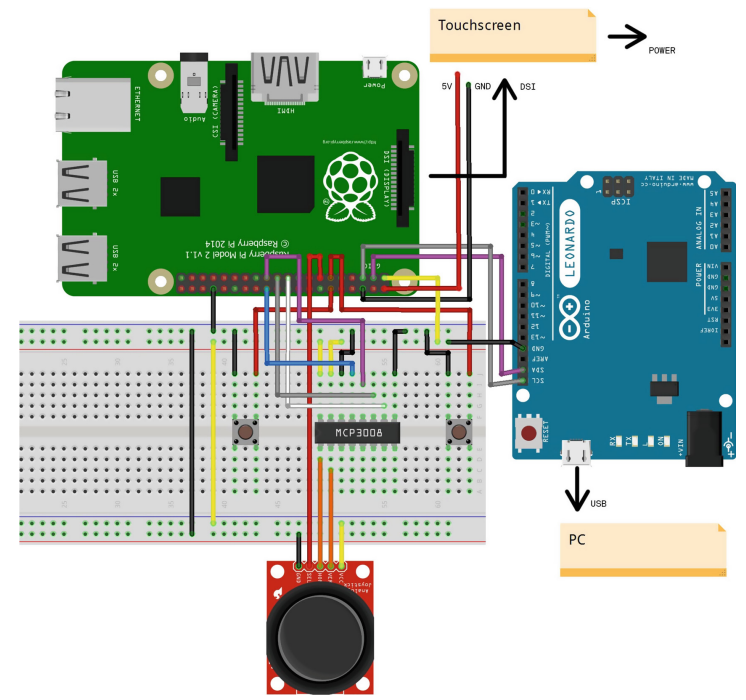

Fig. 14. OHC prototype board scheme. Source: [8].

A picture of the physical prototype we used for experiments can be seen in Fig. 15. Of course, for technical reasons, the physical prototype we built is much thicker than the final intended product. Nevertheless, we already planned the hardware disposition for a more refined - and thinner - version. This hardware disposition is visible in Fig. 16.

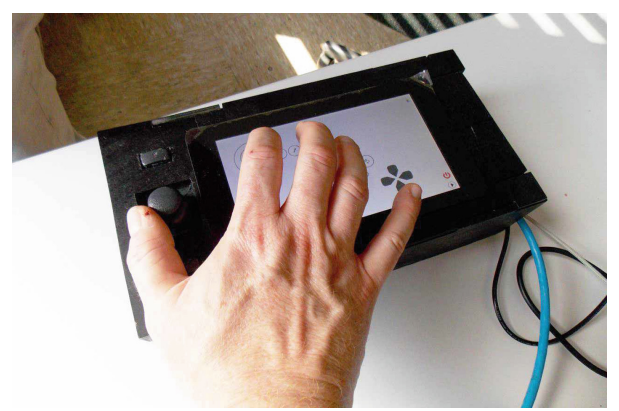

Fig. 15. OHC physical prototype. 


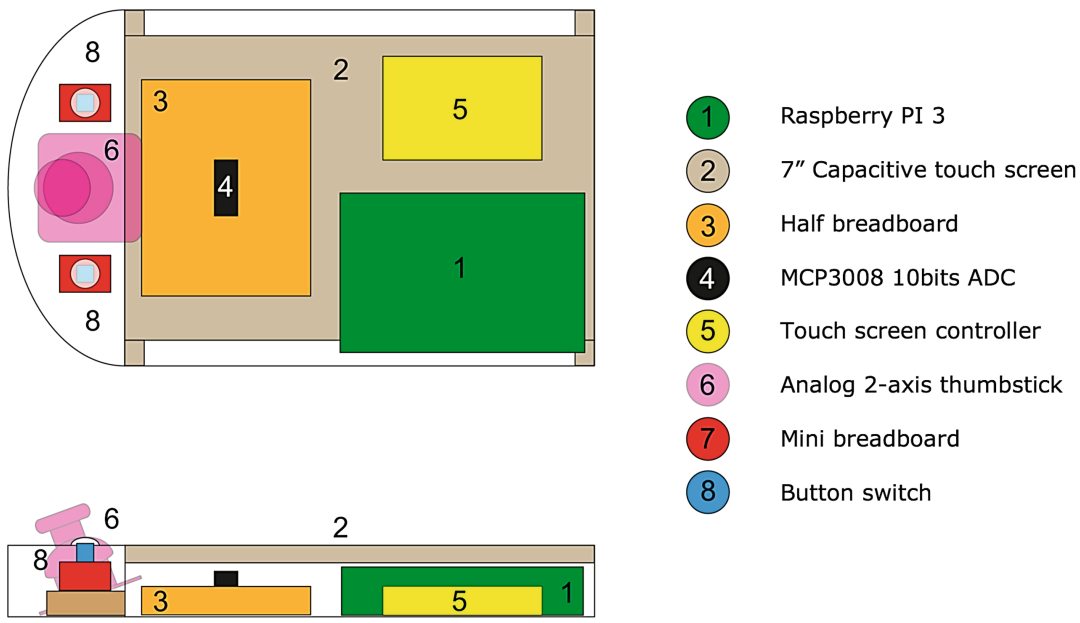

Fig. 16. OHC Hardware disposition for a slimmer version.

\subsection{Software Architecture}

The software architecture is designed to favor the user experience. The main software goal is to virtualize a legacy controller starting from the touchscreen and thumbstick inputs. This task must also be performed with minimal delay.

When first turned on, the OHC software will perform a calibration. As a first step, the user is asked if she wants to (or can) use the buttons and thumbstick. Then, the user will be prompted to lay her hands on the touchscreen in a comfortable position. Starting from the detected touches, a default layout will be proposed, based on the number of available fingers. This layout will be aligned and stretched based on fingers' position. The layout can also be modified later for a better gaming experience.

The internal software supports multiple profiles. This allows several users to share a single device. Moreover, each user can store multiple layouts under her profile. As a result, each player can store a specific layout for each game, depending on personal taste and disability. For severe disabilities, or difficulties in coordinating fingers; macros are supported, and a single tap can be associated to multiple inputs (see also the next section).

The configuration menu can be accessed at any time from the controller itself. The controller touchscreen will provide menus to calibrate, configure controls, and save/load configuration without a requesting additional software on the PC/console (see Fig. 17 for a wireframe and Fig. 18 for a possible configuration sequence). This feature allows $\mathrm{OHC}$ to be more flexible and portable. Currently, there is no direct feedback provided to the game. This means that, during configuration, the game is not going to pause automatically, but it is possible to switch configuration/layout in any game while playing. Switching configuration automatically based on game status is not yet supported.

Finally, OHC can support gestures. Gestures are programmable combinations of command the user can associate to given touch patterns. When enabled, an area of the 


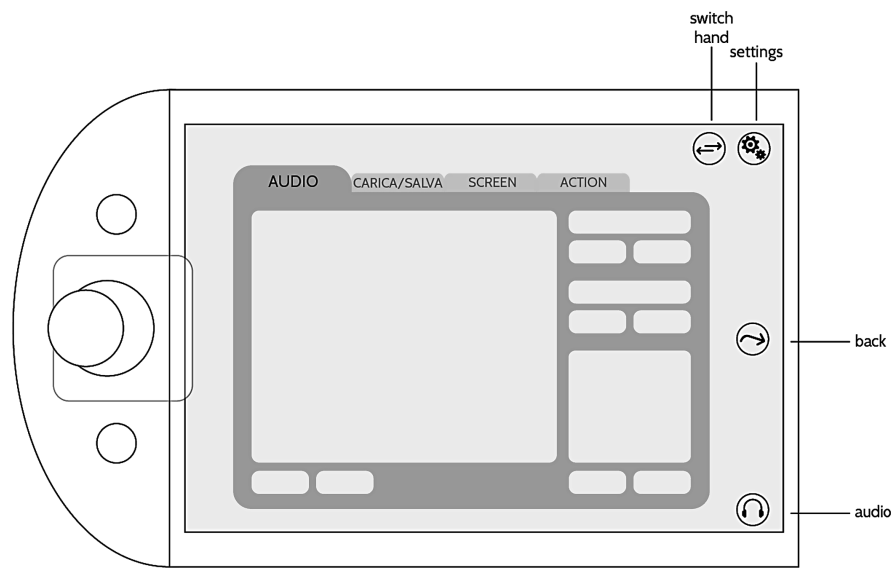

Fig. 17. OHC, Sample menu layout.

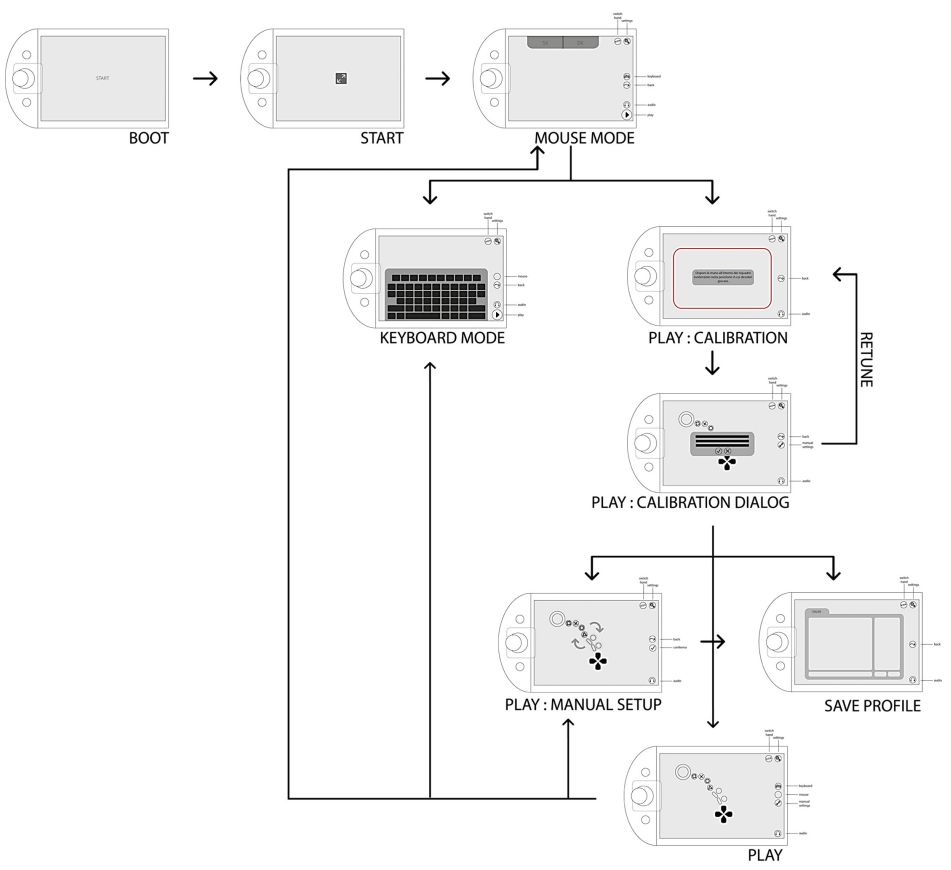

Fig. 18. OHC, sample configuration sequence. 


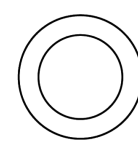

Analog input (virtual thumbstick)
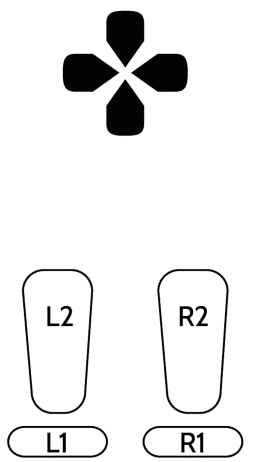

D-Pad input

Secondary

buttons

Fig. 19. Basic GUI Widgets. Source: [8].

touchscreen can be reserved to gesture recognition. Gestures will be detected by the Raspberry PI and translated into a sequence of keystrokes and movements. Currently, we support single, double, and triple touch, rotation, swipes, and two-fingers scrolling.

\subsection{User Interaction}

The Graphical User Interface is the most critical part of the OHC software. As a matter of fact, the GUI must be flexible enough to meet a huge range of user requirements. Moreover, it should also be easy to maintain and extend. For these reasons, we decided to leverage on the computational functionality of Raspberry PI and to implement it using Python and the Kivy framework [19]. Kivy supports the Tangible User Interface Objects (TUIO) paradigm to manage inputs from the touchscreen in a standard way.

The OHC GUI is implemented by composing visual widgets in a hierarchical way. Each widget is taking care of a specific kind of input. An overview of the implemented input widgets is reported in Fig. 19. When performing calibration, one or more widgets are assigned to each finger. Based on selected finger and feedback from the user, the widget will be rotated and stretched to maximize comfort and encompass any movement constraints the gamer may have. Fingers and hand discomfort are reduced by deforming the widget in a way to place each button very close to each finger landing point. Moreover, widgets position should help an easy switch between controls. Figure 20 shows default finger-based transformations applied to primary and secondary buttons.

Nevertheless, additional considerations are required to address missing or not usable fingers. To cope with every possible kind of disability, we designed specific default interfaces for each case. For every variant, a default widget-finger association is proposed, and the actual interface is the result of the deformation and the relocation of widgets basing on the information collected during calibration. Extra care must be devoted to understanding and detecting situations where widgets are too close to each other, hence becoming cumbersome, or, due to mobility constraints on one or more fingers, the widgets position may cause strain. When two widgets are too close the controls may not be effective, especially if the assigned finger has a reduced mobility. 

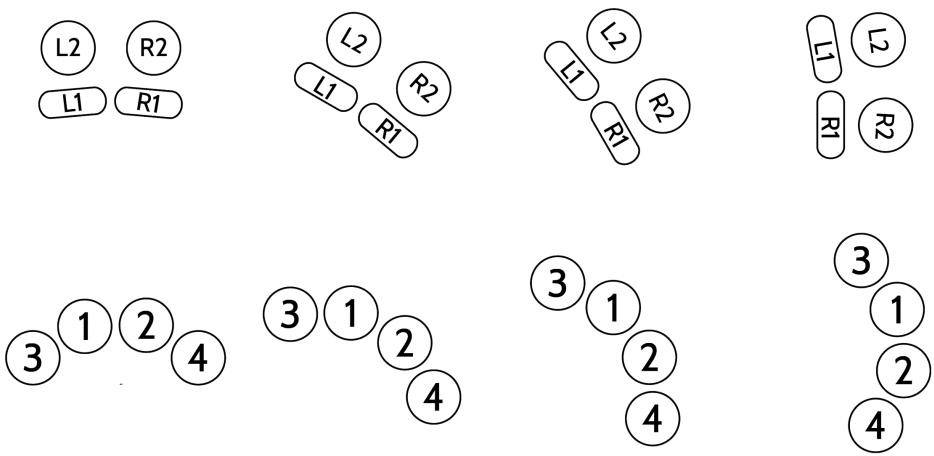

Index $\left(0^{\circ}\right) \quad$ Middle $\left(35^{\circ}\right) \quad \operatorname{Ring}\left(65^{\circ}\right) \quad$ Pinkie $\left(90^{\circ}\right)$

Fig. 20. GUI buttons deformation based on finger. Source: [8].

Strain may be caused by physical conditions, considering that different fingers are sharing muscle and nerve connections. Default interfaces proposed to users having only four or three fingers are reported in Figs. 21 and 22, respectively. In the figures, the outlined areas indicate the working space for the touchscreen, while the large dark dots are fingers positions detected during calibration. The working area and the interface position are calculated starting from these dots.

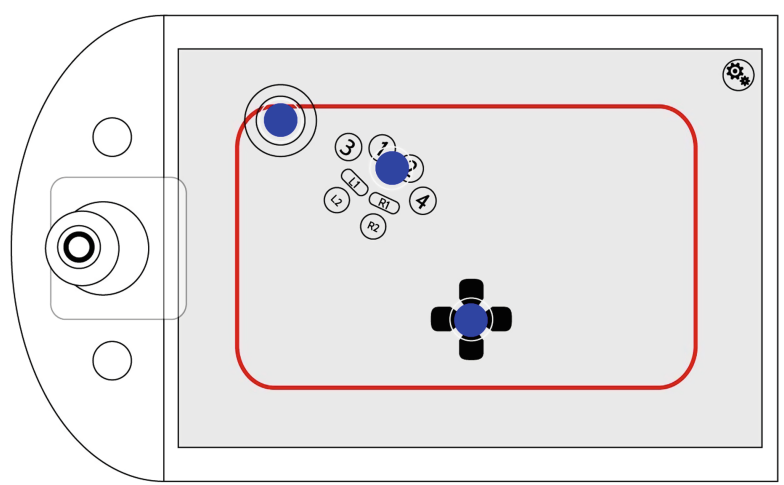

Fig. 21. Default interface for four fingers. Source: [8]. 


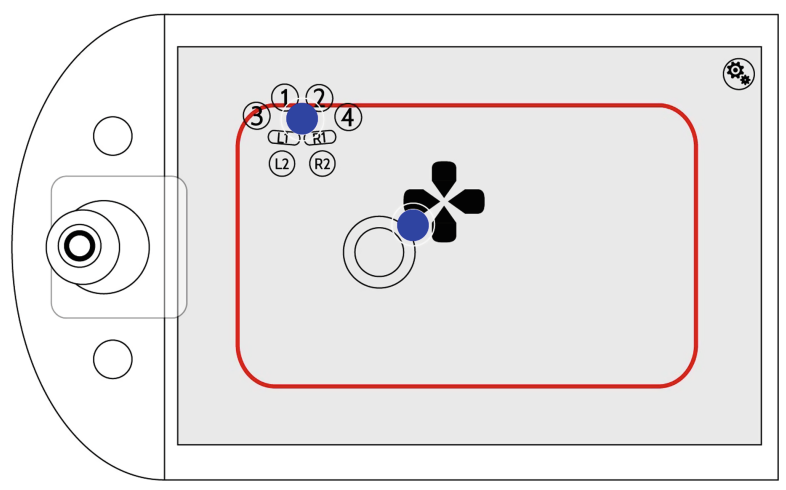

Fig. 22. Default interface for three fingers. Source: [8].

In the case of players having only one finger or missing the hand, the interface must provide a gesture area and should be customized by the user. In this case, we are proposing a dialpad instead of primary and secondary buttons. The result is shown in Fig. 23. This last case is where macros definition can be very useful. Macros can be used to associate a single gesture or a dialed number to complex movements or to a sequence of inputs on a standard controller.

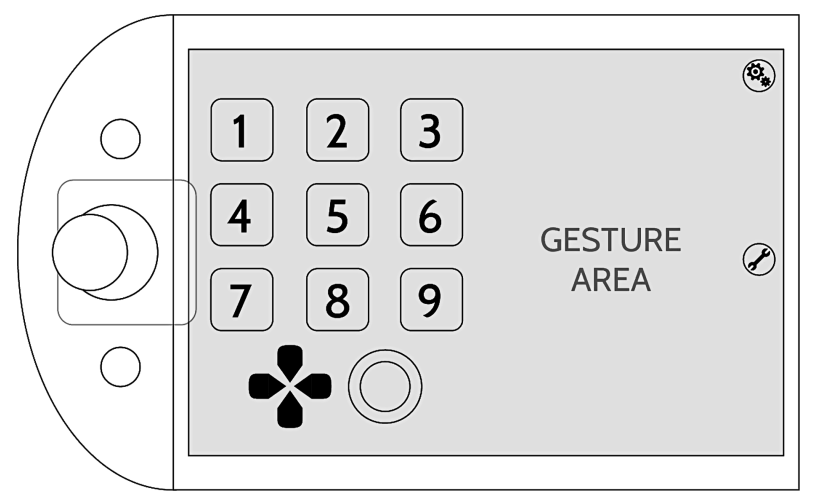

Fig. 23. Default interface for four one finger or no hand. Source: [8].

\subsection{User Acceptance Tests}

To evaluate the user experience while using $\mathrm{OHC}$, we designed an experiment where the participants have been involved in a video games session. This experiment was designed with the single-blind paradigm: a sub-group of participants (test group) have to play the game first with $\mathrm{OHC}$ and then with a commercial gamepad while the other subgroups (control group) have to play the same game reversing the order of the controllers. A feedback form is filled out after each session. The use of both controllers 
is functional to get more information about the comparison of the two interaction models. For the experiments, we leveraged on a level generator for platform games: Fun pledge $2.0[23,27]$. Fun Pledge is platform level generator developed for research purposes where levels layout are generated using a musical base as input. Two levels have been generated; the first has been used as a tutorial while the second was the actual experimental setup.

Procedure. We selected a group of 20 participants with an age between 20 and 40 ( $\mu=25,15$ and $\sigma=3.92$ ) years. The group was composed by 15 men and 5 women. Participants declared to play video games between 1 to $30 \mathrm{~h}$ per week $(\mu=15.65$ and $\sigma=9.53$ ). Albeit we observed a high variability in their adoption of input devices, the majority declared to use a Sony Dualshock or a keyboard with mouse. Only one of the participants used a gamepad designed for people with disability before. All the users used the right hand to interact with the $\mathrm{OHC}$, even if one of them declared to be left handed. For the experiments, we accepted volunteers both with and without physical disabilities in the upper limbs. To people without any disability, we forced a temporary impairment by locking one hand and randomly tying zero, one or two fingers. As a result, the population was divided into three groups: people able to use all the fingers of one hand $(25 \%)$, people able to use 4 fingers (35\%), and people able to use three fingers (40\%).

During the experiments, we arranged sessions with maximum two participants. To each participant, we explained the game mechanics and showed the initial part of the first level (the tutorial). The majority of users were not aware of the proposed game $(80 \%)$ even if $50 \%$ of them claimed to have played similar games. The remaining $20 \%$ already tested the game previously in our lab while performing other research activities $[13,27]$. Anyway, $55 \%$ of declared to enjoy playing platform games, and they rated the game difficulty on a scale from 1 to 10 with rankings from 2 to 8 ( $\mu=5$ and $\sigma=1.65$ ). At the same time, participants evaluated themselves as good players in a range from 4 to $9(\mu=7.05$ and $\sigma=1.39)$ and rated the complexity of game mechanics from 1 to 6 $(\mu=3.2$ and $\sigma=1.58)$.

Each experiment is composed by seven steps as described below.

1. A computer is assigned to the user based on the fact she belongs to the test or control group. This computer is already configured with $\mathrm{OHC}$ or a commercial controller (we used an N30 Pro by 8Bitdo).

2. A first survey is proposed to profile the player.

3. The staff personnel help the user with gamepad setup and configuration and provide general information about the experiment. During this step, the tutorial level is used.

4. The second level is started, and the user must play by herself. This step will be over when the participant either ( $i$ ) completes the level, or ( $i i)$ reaches the game over after 20 lives, or (iii) declares to have fully understood the interaction system with the game.

5. A second survey is proposed about the user experience.

6. If the user still needs to test a second controller, she will be moved to another computer and the protocol is restarted from step number 3 .

7. Otherwise, a final survey is proposed to compare $\mathrm{OHC}$ with the commercial gamepad. 
Experiment Outcome. As already pointed out, we evaluated the experiments by means of surveys. The results presented here are the average of the evaluations provided by the two groups (test and control). For sake of brevity, in the following, we will address the commercial controller simply as "N30". Moreover, all the scores reported below are related to a scale between 1 to 10 .

According to the answers, neither of the controllers seem to cause relevant strain to the hand (3.1 for N30 and 3.7 for $\mathrm{OHC}$ ). Unfortunately, even if users configured the buttons ahead of the game session, they reported a bad configuration for both controllers (4.4 for the test group and 5.8 for the control group). The test group also declared that the $\mathrm{OHC}$ was innovative as a device with a ratio of 6.8 , whereas the control group evaluated the innovation for N30 equal to 4.4.

Do you thing that the features introduced by $\mathrm{OHC}$ are useful?

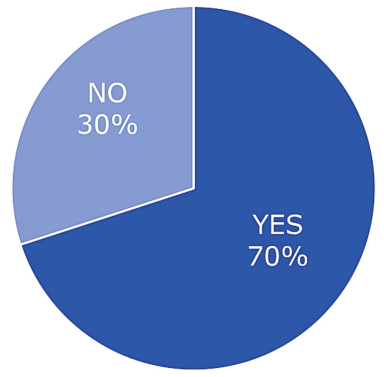

Fig. 24. Feedback about the usefulness of the features introduced by OHC.

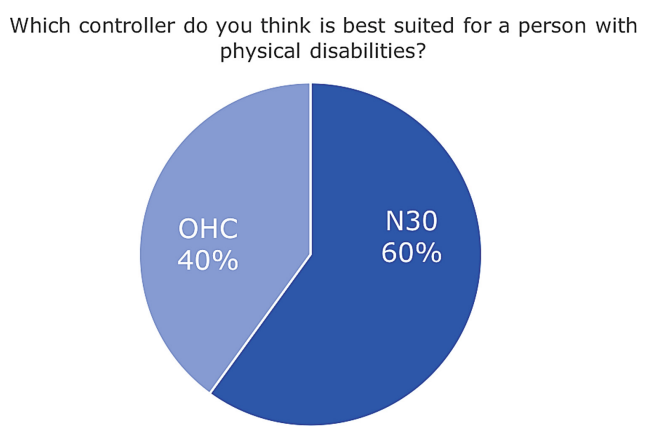

Fig. 25. Feedback about direct comparison between $\mathrm{OHC}$ and $\mathrm{N} 30$.

Specific questions about the design of OHC were also asked only at the test group. They claim that the device configuration was quite easy (6.2), albeit the software interface was not easy enough to understand (5.2). Furthermore, most of the participants evaluated the features introduced by $\mathrm{OHC}$ (e.g., the automatic button calibration using the fingertips) as useful (see Fig. 24). Despite this positive attitude, the general 
mood about how much OHC can help gamers with disabilities is negative, as reported in Fig. 25. Nevertheless, by means of unstructured interviews with the volunteers, it turned out that complaints were more directed toward the manufacturing status of the prototype. Among these complaints, we can mention the distance between thumbstick and display, the buttons size, and the case thickness. These, by the way, are already among the reasons why another hardware prototype is currently in the works (Fig. 16). To better understand this feedback, we can have a look at the answers we received to comparative questions. As a matter of fact, among the users who think that $\mathrm{OHC}$ is not suited for disabled gamers, $90 \%$ is also convinced that the new controller is very innovative (Fig. 26) and has a lot of potential in helping users with physical disabilities (Fig. 27).

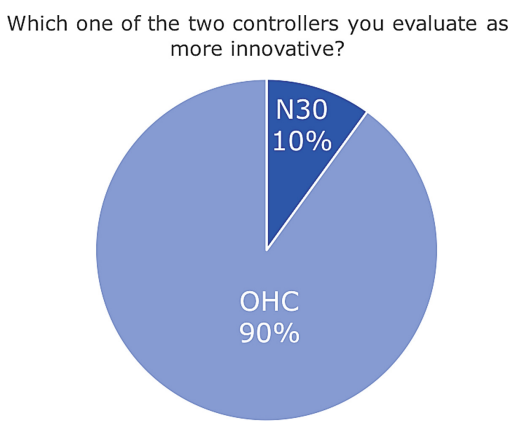

Fig. 26. Feedback about perceived innovation.

Which one of the two controllers do you think has more potential to help people with physical disabilities?

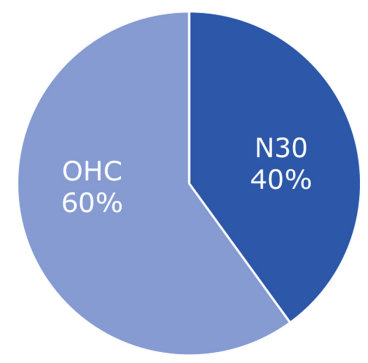

Fig. 27. Feedback about potential usefulness.

\section{Conclusion and Future Work}

In this paper, we discussed about the evolution of gaming control devices and how they try to cope with the requirement of disabled gamers. Our historical analysis seems to hint that mainstream commercial controllers can be exploited in some cases (e.g., a joystick or the Wii Remote Controller) but are not proceeding exactly in the needed 
direction. Nevertheless, many commercial controllers exist - even from first class manufacturer such as Nintendo - that are designed with specific disabilities in mind. By examining the evolution leading to modern controllers and existing solution we can come up with usability guidelines for controllers compatible with physical disabilities. In particular, we used these guidelines to propose OHC (One-Hand Controller). OHC is a gaming controller exploiting a touch surface for easy and fast layout adaptation and a thumbstick to allow exploration of 3D environments. A prototype has been built and tested via a single-blind set of experiments. Results are encouraging in term of potential usefulness but proved also that the first hardware prototype is not up to the task and need refactoring.

In the future, we are planning to finish refactoring the hardware of $\mathrm{OHC}$ to make it thinner and more comfortable to use. Moreover, we must arrange an additional round of experiments to test user acceptance with actually impaired gamers. Another possible improvement will be relative to the interface. The interface will be reworked from a graphical standpoint and a better widgets placement will be devised maybe considering the FFitts [sic] law [1].

\section{References}

1. Bi, X., Li, Y., Zhai, S.: FFitts law: modeling finger touch with fitts' law. In: Proceedings of the SIGCHI Conference on Human Factors in Computing Systems (CHI 2013), pp. 13631372. ACM (2013)

2. Baranyi, R., Willinger, R., Lederer, N., Grechenig, T., Schramm, W.: Chances for serious games in rehabilitation of stroke patients on the example of utilizing the Wii Fit Balance Board. In: IEEE 2nd International Conference on Serious Games and Applications for Health (SeGAH), pp. 1-7 (2013)

3. Barlet, C.M., Spohn, S.D.: Includification: A Practical Guite to Game Accessibility. The Able Gamers Foundation, Charles Town (2012). ISBN-13: 978-1479289356

4. Brown, M., Kehoe, A., Kirakowski, J., Pitt, I.: Beyond the gamepad: HCI and game controller design and evaluation. In: Bernhaupt, R. (ed.) Game User Experience Evaluation. HIS, pp. 263-285. Springer, Cham (2015). https://doi.org/10.1007/978-3-319-15985-0_12

5. Commings, A.H.: The evolution of game controllers and control schemes and their effect on their games. In: 17th Annual University of Southampton Multimedia Systems Conference, vol. 21 (2007)

6. D’Aquaro, N., Maggiorini, D., Mancuso, G., Ripamonti, L.A.: Videogames and elders: a new path in LCT? In: Duffy, V.G. (ed.) ICDHM 2011. LNCS, vol. 6777, pp. 245-254. Springer, Heidelberg (2011). https://doi.org/10.1007/978-3-642-21799-9_28

7. Fanucci, L., Iacopetti, F., Roncella, R.: A console interface for game accessibility to people with motor impairments. In: IEEE International Conference on Consumer Electronics-Berlin (ICCE-Berlin), pp. 206-210 (2011)

8. Gadia, D., Granato, M., Maggiorini, D., Marras, M., Ripamonti, L.A.: A touch-based configurable gamepad for gamers with physical disabilities. In: Proceedings of International Conference on Computer-Human Interaction Research and Application (CHIRA 2017), pp. 67-74 (2017)

9. Game Accessibility Guidelines web page. http://gameaccessibilityguidelines.com/. Accessed 12 Apr 2018 
10. Gordon, C., Roopchand-Martin, S., Gregg, A.: Potential of the Nintendo Wii as a rehabilitation tool for children with cerebral palsy in a developing country: a pilot study. Physiotherapy (United Kingdom) 98(3), 238-242 (2012)

11. Graetz, J.M.: The origin of spacewar. Creative Computing Issue 39 (1981)

12. Grammenos, D., Savidis, A., Stephanidis, C.: Designing universally accessible games. Comput. Entertain. 7(1), 29 (2009)

13. Granato, M., Gadia, D., Maggiorini, D., Ripamonti, L.A.: Emotions detection through the analysis of physiological information during video games fruition. In: Dias, J., Santos, P.A., Veltkamp, R.C. (eds.) GALA 2017. LNCS, vol. 10653, pp. 197-207. Springer, Cham (2017). https://doi.org/10.1007/978-3-319-71940-5_18

14. Hernandez, H.A., Ye, Z., Graham, T.C., Fehlings, D., Switzer, L.: Designing action-based exergames for children with cerebral palsy. In: Proceedings of the SIGCHI Conference on Human Factors in Computing Systems (CHI), pp. 1261-1270 (2013)

15. Iacopetti, F., Fanucci, L., Roncella, R., Giusti, D., Scebba, A.: Game console controller interface for people with disability. In: International Conference on Complex, Intelligent and Software Intensive Systems, pp. 757-762 (2008)

16. IGDA Game Accessibility page. https://igda-gasig.org/about-game-accessibility/. Accessed 12 Apr 2018

17. Kawala-Janik, A., Podpora, M., Gardecki, A., Czuczwara, W., Baranowski, J., Bauer, W.: Game controller based on biomedical signals. In: Proceedings 20th International Conference on Methods and Models in Automation and Robotics (MMAR), pp. 934-939 (2015)

18. Kennedy, M.W., Schmiedeler, J.P., Crowell, C.R., Villano, M., Striegel, A.D., Kuitse, J.: Enhanced feedback in balance rehabilitation using the Nintendo Wii Balance Board. In: 13th International Conference on e-Health Networking, Applications and Services, pp. 162-168 (2011)

19. Kivy homepage. http://www.kivy.org/. Accessed 12 Apr 2018

20. Lopetegui, E., Zapirain, B.G., Mendez, A.: Tennis computer game with brain control using EEG signals. In: 16th International Conference on Computer Games (CGAMES), pp. 228 234 (2011)

21. Maggiorini, D., Ripamonti, L.A., Scambia, A.: Videogame technology to support seniors. In: Proceedings International ICST Conference on Simulation Tools and Techniques (SIMUTOOLS 2012) (2012)

22. Maggiorini, D., Ripamonti, L.A., Zanon, E.: Supporting seniors rehabilitation through videogame technology: a distributed approach. In: Proceedings International Workshop on Games and Software Engineering (GAS 2012) (2012)

23. Mazza, C., Ripamonti, L.A., Maggiorini, D., Gadia, D.: FUN PLEdGE 2.0: a FUNny Platformers LEvels GEnerator (Rhythm Based). In: Proceedings of the 12th Biannual Conference on Italian SIGCHI Chapter (CHItaly 2017), Article 22, 9 p. (2017)

24. Ossmann, R., Miesenberger, K.: Guidelines for the development of accessible computer games. In: Miesenberger, K., Klaus, J., Zagler, W.L., Karshmer, A.I. (eds.) ICCHP 2006. LNCS, vol. 4061, pp. 403-406. Springer, Heidelberg (2006). https://doi.org/10.1007/ 11788713_60

25. Ossmann, R., Miesenberger, K., Archambault, D.: A computer game designed for all. In: Miesenberger, K., Klaus, J., Zagler, W., Karshmer, A. (eds.) ICCHP 2008. LNCS, vol. 5105, pp. 585-592. Springer, Heidelberg (2008). https://doi.org/10.1007/978-3-540-70540-6_83

26. Plunkett, L.: The Disabled-Friendly NES Controller From The 1980's. On Kotaku (online) https://kotaku.com/5241760/. Accessed 12 Apr 2018

27. Ripamonti, L.A., Mannalà, M., Gadia, D., Maggiorini, D.: Procedural content generation for platformers: designing and testing FUN PLEdGE. Multimed. Tools Appl. 76(4), 5001-5050 (2017) 
28. Rowland, J.L., Malone, L.A., Fidopiastis, C.M., Padalabalanarayanan, S., Thirumalai, M., Rimmer, J.H.: Perspectives on active video gaming as a new frontier in accessible physical activity for youth with physical disabilities. Phys. Ther. 96(4), 521-532 (2016)

29. Tollefsen, M., Lunde, M.: Entertaining software for young persons with disabilities. In: Miesenberger, K., Klaus, J., Zagler, W.L., Burger, D. (eds.) ICCHP 2004. LNCS, vol. 3118 , pp. 240-247. Springer, Heidelberg (2004). https://doi.org/10.1007/978-3-540-27817-7_37

30. Tsekleves, E., Paraskevopoulos, I.T., Warland, A., Kilbride, C.: Development and preliminary evaluation of a novel low cost VR-based upper limb stroke rehabilitation platform using Wii technology. Disabil. Rehabil.: Assist. Technol. 11(5), 413-422 (2016)

31. Villar, N., Gilleade, K.M., Ramdunyellis, D., Gellersen, H.: The VoodooIO gaming kit: a real-time adaptable gaming controller. Comput. Entertain. 5(3), 16 (2007)

32. Watanabe, M., Yamamoto, T., Kambara, H., Koike, Y.: Evaluation of a game controller using human stiffness estimated from electromyogram. In: Proceedings of the 2010 Annual International Conference of the IEEE Engineering in Medicine and Biology, pp. 4626-4631 (2010)

33. Westin, T., Bierre, K., Gramenos, D., Hinn, M.: Advances in game accessibility from 2005 to 2010. In: Stephanidis, C. (ed.) UAHCI 2011. LNCS, vol. 6766, pp. 400-409. Springer, Heidelberg (2011). https://doi.org/10.1007/978-3-642-21663-3_43

34. Yuan, B., Folmer, E., Harris, F.C.: Game accessibility: a survey. Univ. Access Inf. Soc. 10(1), 81-100 (2011) 